\title{
Biological Paternal Aunt
}

National Cancer Institute

\section{Source}

National Cancer Institute. Biological Paternal Aunt. NCI Thesaurus. Code C96581.

A female relative who is a sibling of the biological father, and who both share a common ancestor. 